\title{
Vibrational investigation of nucleobases by means of divide and conquer semiclassical dynamics
}

Cite as: J. Chem. Phys. 150, 224107 (2019); https://doi.org/10.1063/1.5100503

Submitted: 17 April 2019 . Accepted: 22 May 2019 . Published Online: 12 June 2019

Fabio Gabas (D), Giovanni Di Liberto (D), and Michele Ceotto (D)

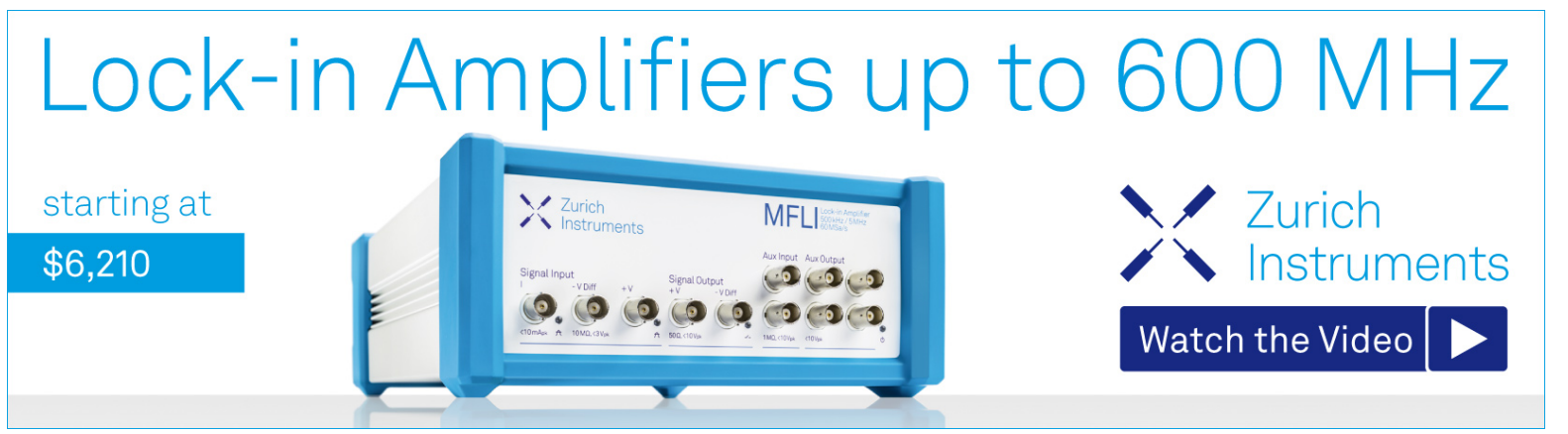




\title{
Vibrational investigation of nucleobases by means of divide and conquer semiclassical dynamics
}

\author{
Cite as: J. Chem. Phys. 150, 224107 (2019); doi: 10.1063/1.5100503 \\ Submitted: 17 April 2019 • Accepted: 22 May 2019 • \\ Published Online: 12 June 2019
}

Fabio Gabas, (D) Giovanni Di Liberto, (D) and Michele Ceotto ${ }^{\text {a) }}$ (D)

AFFILIATIONS

Dipartimento di Chimica, Università degli Studi di Milano, via C. Golgi 19, 20133 Milano, Italy

a) michele.ceotto@unimi.it

\begin{abstract}
In this work, we report a computational study of the vibrational features of four different nucleobases employing the divide-and-conquer semiclassical initial value representation molecular dynamics method. Calculations are performed on uracil, cytosine, thymine, and adenine. Results show that the overall accuracy with respect to experiments is within 20 wavenumbers, regardless of the dimensionality of the nucleobase. Vibrational estimates are accurate even in the complex case of cytosine, where two relevant conformers are taken into account. These results are promising in the perspective of future studies on more complex systems, such as nucleotides or nucleobase pairs.
\end{abstract}

Published under license by AIP Publishing. https://doi.org/10.1063/1.5100503

\section{INTRODUCTION}

The nucleobases adenine (A), cytosine $(\mathrm{C})$, guanine $(\mathrm{G})$, thymine $(\mathrm{T})$, and uracil $(\mathrm{U})$ represent the inner part of nucleotides that are the building blocks of the nucleic acids, DNA and RNA. More specifically, a nucleotide chain constitutes a single strand that interacts with another strand through the bases. These strands compose the secondary and tertiary structures of the nucleic acids as, for example, the famous double helix discovered by Watson and Crick. The interaction between nucleobases follows a specific pairing: A and $\mathrm{G}$, classified as purines, interact, respectively, with $\mathrm{T}$ (for DNA) or $\mathrm{U}$ (for RNA) and $\mathrm{C}$, called pyrimidines. The nucleobases' structure is particularly relevant since it is directly linked to their functionality. ${ }^{2}$ When some of them exist in tautomeric forms, only one conformation is predominant in nature. Also, different tautomers can bring to mispairings between pyrimidines and purines, leading to phenomena known as mutagenesis. ${ }^{1-6}$ For these reasons, an accurate investigation of the structure and properties of all the nucleobase conformations is particularly important. Clearly, detailed studies in the condensed phase are the final goal to understand these properties. ${ }^{7-13}$ However, a preliminary investigation in vacuum is a mandatory step in order to have a spectroscopic clear picture and to be able to discriminate between more complex condensed phase interactions. Specifically, in the gas phase, ${ }^{14}$ it is possible to study the intrinsic properties of such molecules without any intermolecular interaction. Besides, the deep knowledge of biomolecules and their ionic behavior in vacuum is important also for a better understanding and designing of various spectroscopy techniques, such as mass and vibrational spectroscopy. Thus, gas-phase vibrational spectroscopy can certainly help in the characterization and fundamental understanding of nucleobases.

Over the past decades, a lot of experimental work has been done in this direction, starting from the measurement of accurate spectra for adenine, thymine, and uracil, ${ }^{15-22}$ and going to the more complex spectra of cytosine and guanine, which show more than a single relevant isomer, due to the facile tautomerism. ${ }^{23-29}$ These experimental spectra have been often combined with theoretical calculations for a better interpretation. However, given the molecular size of these systems, in most cases, the theoretical prediction was provided by harmonic frequencies calculated at the equilibrium geometry. Even if this approach is computationally cheap and relatively immediate to apply, it neglects all possible resonances and anharmonicities of the potential. Recently, very detailed vibrational studies of uracil have been presented using the canonical van Vleck second-order vibrational perturbation theory (CVPT2), the fully automated generalized second-order vibrational perturbation (GVPT2) approach, and the Hierarchical Intertwined Reduced-Rank Block Power Method (HIRRBPM), obtaining very accurate results compared to the experiment. ${ }^{30-32}$ Also, Perturbation Theory (PT2) has been successful for 
adenine $^{33}$ and for the oxo isomer of citosine. ${ }^{34}$ However, to the best of our knowledge, in the case of thymine, a complete anharmonic computational spectrum is still missing.

In this work, we present a vibrational spectroscopic study of four nucleobases together with their eventual principal isomers, by means of the semiclassical initial value representation (SCIVR) molecular dynamics. ${ }^{35-49}$ SCIVR has been proved in recent years to be very powerful for spectroscopic calculations. $^{50-64}$ It does not suffer from Zero Point Energy Leakage (ZPEL), ${ }^{65}$ and it can be employed for vibrational eigenfunction calculations. ${ }^{66-68}$ In particular, the divide-and-conquer semiclassical initial value representation (DC SCIVR) method for vibrational spectroscopy has been employed reliably in several applications, allowing to obtain semiclassical vibrational spectra of variously sized molecules without any relevant loss of accuracy, and with an average deviation of 20 wavenumbers from either exact or experimental results. ${ }^{69,70}$ Specifically, DC SCIVR has been applied to study challenging high-dimensional and complex systems such as fullerene, supramolecular glycine-based molecules, and water clusters. ${ }^{69,71,72}$

In the present work, we calculate power spectra by performing $a b$ initio molecular dynamics simulations, which has been widely and successfully employed previously for full-dimensional on-thefly semiclassical applications. ${ }^{53-55,72,73}$ More specifically, we want to provide not only an extensive vibrational study of nucleobases but also to open the route to $a b$ initio DC SCIVR calculations on DNA-related molecules. For these goals, our quantum mechanical vibrational estimates are compared with both experiments and other theoretical calculations. These calculations will check and prove DC SCIVR method feasibility and reliability and provide the confidence for future calculations of increasing dimensionality up to pairs of bases and higher dimensional sequences. ${ }^{74-77}$ Here, we investigate four of the five nucleobases, i.e., uracil, cytosine, thymine, and adenine. Since gas-phase spectra of the remaining nucleobase, guanine, are given by a combination of its four most stable conformers and their signals are all within few wavenumbers, guanine is not a good benchmark for testing the accuracy of our method and it has not been investigated.

The paper is organized as follows. Section II recalls the DC SCIVR method for vibrational spectra calculations, together with the computational setup. In Sec. III, we present our results about the nucleobases and we compare them to different experiments and other theoretical calculations. Finally, Sec. IV provides some conclusions and future perspectives.

\section{DIVIDE AND CONQUER SEMICLASSICAL DYNAMICS}

Vibrational power spectra are obtained via Fourier transform of the semiclassical approximation to the survival amplitude $\left\langle\chi \mid \chi_{t}\right\rangle$,

$$
I(E)=\frac{1}{2 \pi \hbar} \int d t e^{\frac{i}{\hbar} E t}\left\langle\chi\left|e^{-\frac{i}{\hbar} \hat{H} t}\right| \chi\right\rangle,
$$

where $|\chi\rangle$ is a given reference state, $\hat{H}$ is the Hamiltonian operator, and $\exp [-i \hat{H} t / \hbar]$ is the quantum mechanical time-evolution operator. We calculate this propagator using the semiclassical approximation.
Semiclassical theory takes advantage of the Feynman path integral representation of the quantum propagator, ${ }^{79}$ in which a quantum mechanical amplitude, describing the probability for a particle of mass $m$ to move from a certain initial state $\mathbf{q}(0)$ to a final one $\mathbf{q}(t)$ at time $t$, is calculated as

$$
\left\langle\mathbf{q}(t)\left|e^{-\frac{i}{h} \hat{H} t}\right| \mathbf{q}(0)\right\rangle=\sqrt{\left(\frac{m}{2 \pi i \hbar t}\right)^{F}} \int \wp[\mathbf{q}(t)] e^{\frac{i}{\hbar} S_{t}(\mathbf{q}(t), \mathbf{q}(0))},
$$

where the integral of the differential $\wp[\mathbf{q}(t)]$ is given by the summation over all possible paths from $\mathbf{q}(0)$ to $\mathbf{q}(t)$. $S_{t}(\mathbf{q}(t), \mathbf{q}(0))$ is the action of each path, and $F$ is the number of degrees of freedom. When the stationary phase approximation for the path integration is enforced, only classical paths contribute. This approximation leads to the well known van Vleck propagator, ${ }^{80}$ where the integral is replaced by a sum over all classical paths connecting the starting position $\mathbf{q}(0)$ to the ending one $\mathbf{q}(t)$ in an amount of time $t$,

$$
\begin{aligned}
\left\langle\mathbf{q}(t)\left|e^{-\frac{i}{h} \hat{H} t}\right| \mathbf{q}(0)\right\rangle & \sqrt{\left(\frac{1}{2 \pi i \hbar}\right)^{F}} \sum_{\text {Clpaths }} e^{\frac{i}{\hbar} S_{t}(\mathbf{q}(t), \mathbf{q}(0))} \\
& \times\left|\frac{\partial \mathbf{p}(0)}{\partial \mathbf{q}(t)}\right|^{\frac{1}{2}} e^{-i \pi v / 2} .
\end{aligned}
$$

The index $v$ is called Morse, or Maslov index, and it accounts for the sign change of the determinant $\left|\frac{\partial \mathbf{p}(0)}{\partial \mathbf{q}(t)}\right|$. This version of the propagator is quite cumbersome and not practical since it requires dealing with a root search problem with fixed boundary conditions. This issue has been overcome by the initial value representation trick proposed by Miller, ${ }^{37}$ where the integrand of Eq. (1) is written as

$$
\begin{aligned}
\left\langle\chi\left|e^{-\frac{i}{h} \widehat{H} t}\right| \chi\right\rangle & \sqrt{\left(\frac{1}{2 \pi i \hbar}\right)^{F}} \iint d \mathbf{q}(0) d \mathbf{p}(0)\langle\chi \mid \mathbf{q}(t)\rangle\langle\mathbf{q}(0) \mid \chi\rangle \\
& \times\left|\frac{\partial \mathbf{q}(t)}{\partial \mathbf{p}(0)}\right|^{\frac{1}{2}} e^{\frac{i}{\hbar} S_{t}(\mathbf{q}(0), \mathbf{p}(0))} e^{-i \pi v / 2} .
\end{aligned}
$$

In this version of the propagator, the survival amplitude can be obtained by sampling trajectories with different initial conditions $(\mathbf{p}(0), \mathbf{q}(0))$ and it is amenable to the Monte Carlo integration. Later, Heller proposed a very flexible and numerically stable representation of the semiclassical propagator based on coherent states $^{81,82}$ of the type

$$
\langle\mathbf{q} \mid \mathbf{p}(t), \mathbf{q}(t)\rangle=\left(\frac{\operatorname{det}(\boldsymbol{\Gamma})}{\pi^{F}}\right)^{\frac{1}{4}} e^{-\frac{1}{2}(\mathbf{q}-\mathbf{q}(t))^{T} \boldsymbol{\Gamma}(\mathbf{q}-\mathbf{q}(t))+\frac{i}{\hbar} \mathbf{p}^{T}(t)(\mathbf{q}-\mathbf{q}(t))},
$$

where in our simulations, the $\boldsymbol{\Gamma}$ matrix is chosen to be constant in time and diagonal, with elements equal to the normal mode vibrational frequencies. The wavepacket in Eq. (5) is centered at the classical position $\mathbf{q}(t)$ and momentum $\mathbf{p}(t)$, and it follows the classical trajectory. The expression of the quantum propagator in this representation is the well known Heller-Herman-Kluk-Kay (HHKK) propagator $^{83-85}$ 


$$
\begin{aligned}
e^{-\frac{i}{h} \hat{H} t}= & \left(\frac{1}{2 \pi \hbar}\right)^{F} \iint d \mathbf{p}(0) d \mathbf{q}(0) C_{t}(\mathbf{p}(0), \mathbf{q}(0)) e^{\frac{i}{\hbar} S_{t}(\mathbf{p}(0), \mathbf{q}(0))} \\
& \times|\mathbf{p}(t), \mathbf{q}(t)\rangle\langle\mathbf{p}(0), \mathbf{q}(0)|,
\end{aligned}
$$

where $C_{t}(\mathbf{q}(0), \mathbf{p}(0))$ is the pre-exponential factor and it is equal to

$$
C_{t}(\mathbf{q}(0), \mathbf{p}(0))=\sqrt{\operatorname{det}\left[\frac{1}{2}\left(\mathbf{M}_{q q}+\boldsymbol{\Gamma}^{-1} \mathbf{M}_{p p} \boldsymbol{\Gamma}+\frac{i}{\hbar} \boldsymbol{\Gamma}^{-1} \mathbf{M}_{p q}+\frac{\hbar}{i} \mathbf{M}_{q p} \boldsymbol{\Gamma}\right)\right]},
$$

and where $\mathbf{M}_{\mathbf{i j}}$ are the monodromy (or stability) matrix elements defined as $\mathbf{M}_{\mathbf{i j}}=\frac{\partial \mathbf{i}_{\mathbf{t}}}{\partial \mathbf{j}_{0}}$, where $\mathbf{i}_{\mathbf{t}}$ is calculated at time $t$ and $\mathbf{j}_{\mathbf{0}}$ is calculated at time zero. ${ }^{86,87}$ The Monte Carlo integration in Eq. (6) usually needs a high number of trajectories for convergence. For systems having more than a few degrees of freedom, some filtering techniques have been proposed to speed up the convergence. ${ }^{88-91} \mathrm{~A}$ well established procedure is the time-averaging (TA) filtering, in which the semiclassical integrand can be worked out to be positively definite ${ }^{92}$ by taking advantage of the so-called separable approximation of the pre-exponential factor, where only the phase is taken into account, i.e., $C_{t}(\mathbf{q}(0), \mathbf{p}(0)) \sim e^{\frac{i}{\hbar} \varphi_{t}}$ and $\varphi_{t}=$ phase $\left[C_{t}(\mathbf{q}(0), \mathbf{p}(0))\right]$. Using the propagator in Eq. (6), the time-averaging filter and the separable approximation, one obtains the following formula for the spectral density:

$$
\begin{aligned}
I(E)= & \left(\frac{1}{2 \pi \hbar}\right)^{F} \iint d \mathbf{p}(0) d \mathbf{q}(0) \frac{1}{2 \pi \hbar T} \\
& \times\left|\int_{0}^{T} e^{\frac{i}{h}\left[S_{t}(\mathbf{p}(0), \mathbf{q}(0))+E t+\varphi_{t}\right]}\langle\chi \mid \mathbf{p}(t) \mathbf{q}(t)\rangle d t\right|^{2} .
\end{aligned}
$$

Equation (8) has been employed to perform vibrational estimates of small-sized molecules requiring roughly a 1000 of classical trajectories per degree of freedom to converge. ${ }^{93-96}$ Unfortunately when prefitted Potential Energy Surfaces (PESs) are not available, the number of required trajectories is still too computational demanding for on-the-fly or direct dynamics approaches. In this event, only few classical trajectories can be afforded. For these reasons, in recent years, starting from Eq. (8), in our group, the Multiple Coherent State approach (MC SCIVR) has been developed, in which by properly choosing the initial conditions of the classical trajectories and the reference state it is possible to regain spectra with few or even one classical trajectory and retaining the typical semiclassical accuracy of roughly $20 \mathrm{~cm}^{-1}$. In the single trajectory implementation, the initial conditions are chosen as $(\mathbf{p}(0), \mathbf{q}(0))=$ $\left(\mathbf{p}_{e q}, \mathbf{q}_{e q}\right)$, where $\mathbf{p}_{e q}$ and $\mathbf{q}_{e q}$ stand for the coordinates of the reference coherent state $|\chi\rangle$. The phase space integral of Eq. (8) reduces to a single trajectory formulation for each spectroscopic peak of the type

$$
\begin{aligned}
I(E)= & \left(\frac{1}{2 \pi \hbar}\right)^{F} \frac{1}{2 \pi \hbar T} \mid \int_{0}^{T} e^{\frac{i}{\hbar}\left[s_{t}\left(\mathbf{p}_{e q}, \mathbf{q}_{e q}\right)+E t+\varphi_{t}\left(\mathbf{p}_{e q}, \mathbf{q}_{e q}\right)\right]} \\
& \times\left.\langle\chi \mid \mathbf{p}(t), \mathbf{q}(t)\rangle d t\right|^{2},
\end{aligned}
$$

where $\mathbf{q}_{e q}$ is usually chosen to be equal to the equilibrium configuration and $\mathbf{p}_{e q}$ is set in order to provide a total initial kinetic energy equal to the harmonic zero point energy (ZPE), which is distributed as $p_{e q}^{i}=\sqrt{\omega_{i}}$ among mass-scaled vibrational normal modes, where $\omega_{i}$ is the harmonic frequency of the $i$ th mode. $\mathbf{p}(t)$ and $\mathbf{q}(t)$ are the respective time-evolved quantities. Equation (9) is our working equation for MC SCIVR calculation. Moreover, in the MC SCIVR approach, the reference state can be tailored to decompose the spectrum mode by mode. For an $i$ th mode, we have

$$
|\chi\rangle_{i}=\prod_{j=1}^{F}\left[\left|p_{j, e q}^{i}(0), q_{j, e q}^{i}(0)\right\rangle+\varepsilon^{i}\left|-p_{j, e q}^{i}(0), q_{j, e q}^{i}(0)\right\rangle\right],
$$

where the index $j$ runs over the number of vibrational degrees of freedom $\mathrm{F}^{54,97}$ If the $\varepsilon$ vector is set equal to one, then the zero point energy peak is obtained (together with other even peaks), while by setting $\varepsilon^{i}=-1$ only for a certain $i$ th mode, then the $i$ th fundamental excitation will be enhanced, together with the other odd overtones of $i$ th mode. ${ }^{97}$ This tool is particularly helpful in presence of crowded spectra, where peaks are very close in energy, as it commonly happens by increasing the dimensionality of the molecule under exam. This strategy successfully allowed to recover accurate spectra of molecules as complex as glycine.

Unfortunately, MC SCIVR runs out of steam when the system dimensionality overcomes 25-30 degrees of freedom because of the so-called curse of dimensionality problem. Recently, we have addressed this issue by proposing the DC SCIVR method, ${ }^{69,70}$ where full dimensional classical simulations are projected onto subdimensional spaces for semiclassical subdimensional spectroscopic calculations. The same working formula of Eq. (8) is employed but formulated in terms of subspace coordinates. More specifically, the spectral density for a M-dimensional subspace is

$$
\begin{aligned}
\widetilde{I}(E)= & \left(\frac{1}{2 \pi \hbar}\right)^{M} \iint d \tilde{\mathbf{p}}(0) d \tilde{\mathbf{q}}(0) \frac{1}{2 \pi \hbar T} \mid \int_{0}^{T} e^{\frac{i}{\hbar}\left[\tilde{S}_{t}(\tilde{\mathbf{p}}(0), \tilde{\mathbf{q}}(0))+E t+\tilde{\phi}_{t}\right]} \\
& \times\left.\langle\tilde{\chi} \mid \tilde{\mathbf{p}}(t), \tilde{\mathbf{q}}(t)\rangle d t\right|^{2}
\end{aligned}
$$

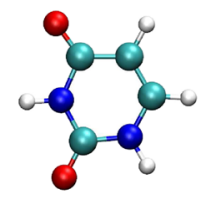

a)

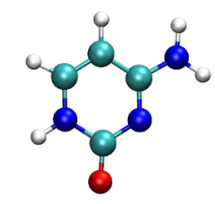

b)

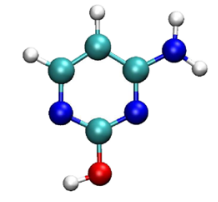

c)

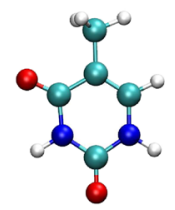

d)

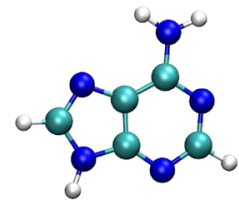

e)
FIG. 1. Molecular structures of the simulated nucleobases. (a) uracil, (b) and (c) respectively, oxocytosine and hydroxycytosine conformers, (d) thymine, and (e) adenine. Red: $\mathrm{O}$, gray: $\mathrm{H}$, blue: $\mathrm{N}$, and dark green: $\mathrm{C}$. 
where $\sim$ denotes the projected quantities. The full-dimensional spectrum is recovered as a combination of reduced dimensionality ones. $\langle\tilde{\mathbf{x}} \mid \tilde{\mathbf{p}} \tilde{\mathbf{q}}\rangle=\prod_{i=1}^{M}\left\langle x_{i} \mid p_{i} q_{i}\right\rangle$ are the projected coherent states written as direct product of monodimensional ones and involving only the degrees of freedom in the subspace. $\tilde{S}_{t}$ is the projected action, and $\tilde{\phi}_{t}$ is the phase of the projected pre-exponential factor. This latter term is obtained from the reduced dimensionality monodromy matrix elements upon a singular value decomposition of the fulldimensional ones. 98,99 The projected action functional is written as

$$
\tilde{S}_{t}(\tilde{\mathbf{p}}(0), \tilde{\mathbf{q}}(0))=\int_{0}^{t}\left[\frac{1}{2} m \tilde{\dot{\mathbf{q}}}^{2}\left(t^{\prime}\right)-V_{S}\left(\tilde{\mathbf{q}}\left(t^{\prime}\right)\right)\right] d t^{\prime},
$$

where the kinetic part is trivially projected since it is naturally separable. For the potential part, we assume that the projected potential depends on the degrees of freedom contained in the subspace and the remaining ones are downgraded to parameters. We include a time-dependent external scalar field $\lambda(t)$ to ensure that the equation of the projected potential is exact in the limit of a separable system and it accounts for the contribution arising from the degrees of freedom not belonging to the subspace,

$$
\begin{aligned}
V_{S}(\tilde{\mathbf{q}}(t))= & V\left(\tilde{\mathbf{q}}(t) ; \mathbf{q}_{N_{v i b}-M}^{e q}\right)+\lambda(t), \\
\lambda(t)= & V\left(\tilde{\mathbf{q}}(t) ; \mathbf{q}_{N_{v i b}-M}(t)\right)-\left[V\left(\tilde{\mathbf{q}}(t) ; \mathbf{q}_{N_{v i b}-M}^{e q}\right)\right. \\
& \left.+V\left(\mathbf{q}_{M}^{e q} ; \mathbf{q}_{N_{v i b}-M}(t)\right)\right] .
\end{aligned}
$$

Clearly, the definition of the subspaces is a critical issue within this approach since it is responsible for the accuracy of the action and the monodromy matrix calculation. As for Eq. (9), Eq. (11)

TABLE I. Vibrational fundamental excitations of uracil. Values are reported in $\mathrm{cm}^{-1}$. The first column reports the label of the excitation, while the second one reports the experimental values from Refs. 18 and 103. Third and fourth columns contain our

\begin{tabular}{|c|c|c|c|c|c|c|c|}
\hline \multirow[b]{2}{*}{ Label } & \multirow[b]{2}{*}{ Exp } & \multirow{2}{*}{$\begin{array}{c}\text { Harmonic } \\
\text { B3LYP/ } \\
\text { aug-cc-pvdz }\end{array}$} & \multirow{2}{*}{ 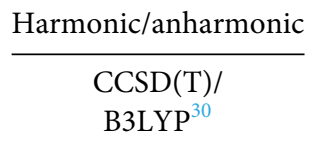 } & \multicolumn{2}{|c|}{ VPT2 } & \multicolumn{2}{|c|}{ Semiclassical } \\
\hline & & & & GVPT2 & CVPT2 & MC SCIVR & DC SCIVR \\
\hline 1 & & 168 & 140 & 132 & 139.7 & 156 & 160 \\
\hline 2 & 185 & 181 & 159 & 155 & 157.9 & 164 & 170 \\
\hline 3 & 391 & 395 & 387 & 386 & 385.6 & 380 & 370 \\
\hline 4 & 411 & 405 & 388 & 384 & 384.4 & 396 & 390 \\
\hline 5 & 516 & 522 & 517 & 510 & 510.8 & 524 & 510 \\
\hline 6 & 537 & 543 & 541 & 530 & 531.1 & 520 & 535 \\
\hline 7 & 562 & 560 & 545 & 549 & 535.3 & 552 & 550 \\
\hline 8 & 551 & 582 & 559 & 555 & 549.4 & 544 & 560 \\
\hline 9 & 662 & 698 & 670 & 654 & 651.4 & 676 & 680 \\
\hline 10 & 718 & 729 & 728 & 711 & 715.8 & 708 & 710 \\
\hline 11 & 757 & 764 & 765 & 746 & 756.1 & 680 & 750 \\
\hline 12 & 759 & 771 & 773 & 751 & 751.8 & 748 & 750 \\
\hline 13 & 804 & 821 & 814 & 793 & 803.2 & 788 & 820 \\
\hline 14 & 987 & 963 & 973 & 954 & 955.9 & 940 & 940 \\
\hline 15 & 958 & 966 & 968 & 940 & 947.5 & 952 & 950 \\
\hline 16 & 980 & 988 & 995 & 978 & 979.9 & 968 & 970 \\
\hline 17 & 1075 & 1083 & 1084 & 1061 & 1060.8 & 1052 & 1060 \\
\hline 18 & 1185 & 1194 & 1205 & 1176 & 1179.9 & 1104 & 1200 \\
\hline 19 & 1217 & 1222 & 1248 & 1221 & 1214.4 & 1208 & 1200 \\
\hline 20 & 1359 & 1379 & 1394 & 1384 & 1382.0 & 1336 & 1340 \\
\hline 21 & 1389 & 1402 & 1414 & 1355 & 1351.0 & 1340 & 1340 \\
\hline 22 & 1400 & 1412 & 1427 & 1388 & 1394.1 & 1376 & 1370 \\
\hline 23 & 1472 & 1497 & 1505 & 1466 & 1462.7 & 1464 & 1460 \\
\hline 24 & 1643 & 1673 & 1678 & 1643 & 1642.8 & 1644 & 1630 \\
\hline 25 & 1706 & 1757 & 1762 & 1733 & 1729.5 & 1720 & 1720 \\
\hline 26 & 1764 & 1788 & 1790 & 1761 & 1761.2 & 1764 & 1760 \\
\hline 27 & & 3210 & 3218 & 3072 & 3081.0 & 2980 & 3070 \\
\hline 28 & & 3250 & 3253 & 3117 & 3133.6 & 3144 & 3160 \\
\hline 29 & 3435 & 3585 & 3602 & 3436 & 3435.2 & 3480 & 3510 \\
\hline 30 & 3485 & 3631 & 3653 & 3485 & 3486.3 & 3536 & 3550 \\
\hline MAE & & 26 & 30 & 10 & 9 & 22 & 20 \\
\hline
\end{tabular}
harmonic estimates and the hybrid harmonic/anharmonic CCSD(T)/B3LYP values, respectively. GVPT2 and CVPT2 estimates of Refs. 30 and 31 are reported under the VPT2 columns. The last two columns report the energy levels calculated with MC SCIVR and DC SCIVR. "MAE" stands for Mean Absolute Error. 
can be reduced to a single trajectory formulation and this formulation will be employed in our calculations. Next, one desires to group in the same subspace the most interacting degrees of freedom. We described several strategies in Ref. 70. Here, we employ the one based on the Hessian matrix since the off-diagonal terms indicate the level of coupling between degrees of freedom. The procedure to separate the full-dimensional space starts by defining a time-averaged Hessian matrix $\mathbf{H}$ along a test trajectory, where $\bar{H}_{i j}=\frac{1}{N_{\text {steps }}} \sum_{i=1}^{N_{\text {steps }}} H_{i j} .{ }^{69,70}$ We fix a threshold parameter that is comparable with the normal mode mass-scaled off-diagonal terms of $\overline{\boldsymbol{H}}$. If $\bar{H}_{i j} \geq \varepsilon$, then the level of coupling is considered significant and the degrees of freedom are enrolled in the same subspace. Instead, if $\bar{H}_{i j} \leq \varepsilon, i$ and $j$ modes are on different subspaces, unless there exists a third mode $k$ such that $\bar{H}_{k i} \geq \varepsilon$ and/or $\bar{H}_{k j} \geq \varepsilon$. The best possible scenario is when there are few subspaces and they are big enough to retain most of the couplings. However, in practice, the threshold choice is often a trade-off between accuracy and feasibility of the semiclassical calculation.

Molecular dynamics simulations in this work are performed using the NWChem package ${ }^{100}$ at a density functional theory (DFT) B3LYP/aug-cc-pvdz level of theory, ${ }^{101}$ which is a typical setup for semiclassical $a b$ initio calculations and represents a good tradeoff between accuracy and computational overhead. For each conformer, we evolve a single trajectory for a total of 25000 a.u., starting from $\left(\mathbf{p}_{e q}, \mathbf{q}_{e q}\right)$, where $\mathbf{q}_{e q}$ is the equilibrium configuration and $\mathbf{p}_{e q}$ is the momenta vector, setting each momentum to have a total initial energy equal to the harmonic ZPE of the molecule. With the exception of the uracil molecule, we calculate the Hessian every two steps and approximate it otherwise. ${ }^{86,87}$ This is a typical setup that is often enough to recover MC SCIVR and DC SCIVR vibrational spectra with an average accuracy within 20-25 wavenumbers. $53,73,96,102$

\section{RESULTS AND DISCUSSION}

We start our investigation with the lowest dimension nucleobase, i.e., uracil. For this system, we perform both MC SCIVR and DC SCIVR calculations to prove once more the reliability of the DC SCIVR method. Results are compared with experiments and high level VPT2 calculations. Then, we move to cytosine, for which there are two conformers which are spectroscopic relevant, and to thymine and adenine vibrational spectra and compare them to the available experimental results. With the exception of uracil, full-dimensional MC SCIVR calculations cannot be afforded for the other nucleobases. In these cases, only DC SCIVR simulations will be performed. The molecular structures of these molecules are reported in Fig. 1.

\section{A. Uracil}

Uracil is made of one pyrimidine ring resulting into 12 atoms. The molecular structure of the global minimum is reported in panel (a) of Fig. 1. For this molecular system, the energy difference between the global minimum (oxo form) and its tautomer (hydroxy form) is around $45 \mathrm{~kJ} / \mathrm{mol}^{21}$ For this reason, we perform our semiclassical study only on the structure reported in panel (a) of Fig. 1 since it is expected to be by far the most representative one. In the past years, this molecule was the subject of several studies, both with experimental and theoretical approaches. Specifically, we will compare our semiclassical vibrational estimates with experimental values $^{18,103,104}$ and GVPT2 and CVPT2 calculated energy levels. ${ }^{30,31}$ As a first evaluation of the level of theory, we report in Table I the computed harmonic frequencies of the molecule at B3LYP/aug-ccpvdz level of theory together with those of Ref. 30, calculated using a hybrid CCSD(T)/B3LYP force field, where harmonic CCSD(T) estimates have been corrected using GVPT2 estimates at the level

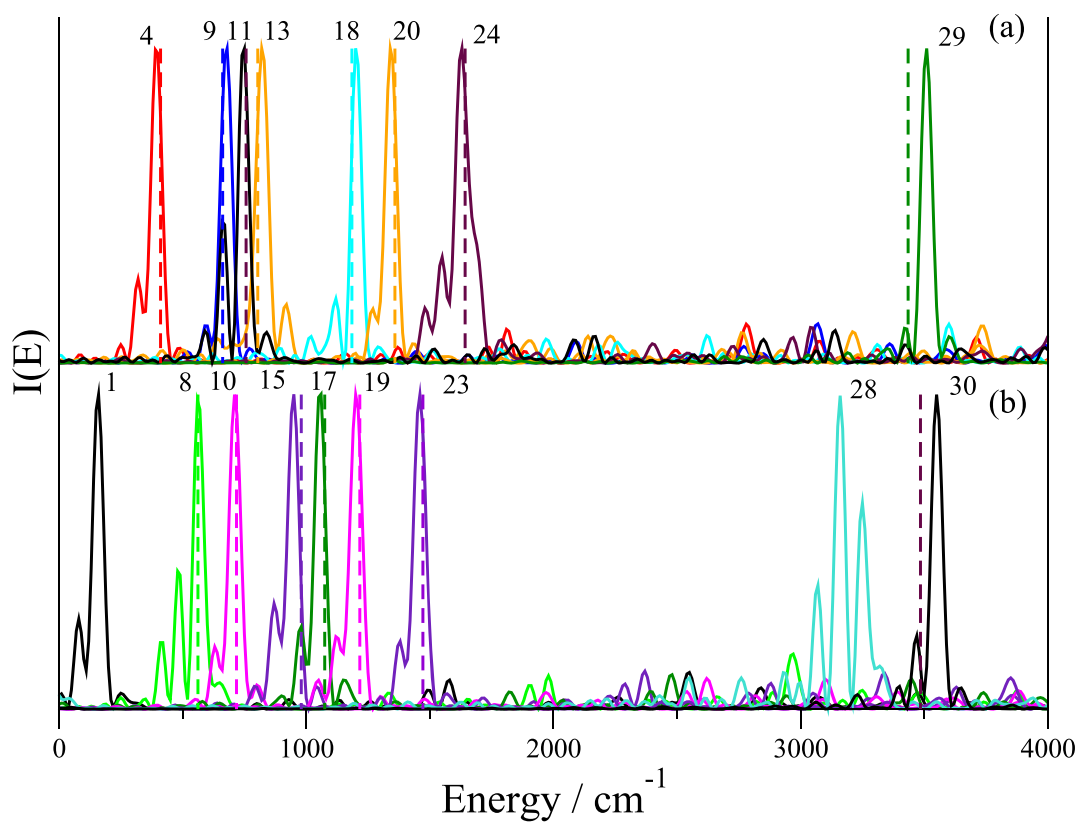

FIG. 2. Panel (a) and (b): DC SCIVR fundamental energy levels respect to the ZPE of the 17-dimensional subspace. Each fundamental excitation was obtained by tailoring the reference state $|\chi\rangle$ according to Eq. (10). The labels of the energy levels are given according to the nomenclature reported in Table Vertical lines are centered at experimental values. 
of B3LYP theory for anharmonicities. We can observe a good agreement between these two column values, suggesting that our computational setup is a good harmonic estimate for a possible accuracy and feasibility of the semiclassical simulations. When moving to the semiclassical dynamics results, each uracil fundamental frequency is evaluated by tailoring the reference state of the semiclassical integrand according to the MC SCIVR approach of Eq. (10). Given the above mentioned dimensional limits, uracil represents also a good benchmark for comparison between MC SCIVR and DC SCIVR. The full-dimensional space is partitioned employing the Hessian matrix method. ${ }^{69}$ A threshold value equal to $\varepsilon=4 \times 10^{-7}$ leads to a 17-dimensional subspace, and all other subspaces are monodimensional. In Table I, the computed MC SCIVR and DC SCIVR energy levels are reported. In the fifth and sixth columns of the same table, the GVPT2 and CVPT2 estimates are slightly more accurate than the semiclassical ones probably because of the higher level of $a b$ initio theory employed.

TABLE II. Vibrational fundamental excitations of the oxocytosine isomer. Values are reported in $\mathrm{cm}^{-1}$. The first column reports the label of the excitation, and the second and the third ones report the experimental values taken from Table III of Ref. 23 measured in Ne and Ar matrices, respectively. Fourth column is for VPT2 energy levels. ${ }^{34}$ Fifth column is for Harmonic results using a B3LYP/aug-cc-pvdz level of theory, and the last column reports the energy levels calculated with DC SCIVR at the same level of theory. The MAEs of DC SCIVR with respect to both experiments is reported into the last row, where the value in parentheses is for the comparison with the experimental values in the Ar matrix.

\begin{tabular}{|c|c|c|c|c|c|}
\hline Label & $\mathrm{Exp} / \mathrm{Ne}$ & Exp/Ar & VPT2 & Harmonic & DC SCIVR \\
\hline 8 & & & & 546 & 520 \\
\hline 9 & 571 & 575 & & 578 & 560 \\
\hline 10 & 614 & 614 & & 643 & 630 \\
\hline 11 & 717 & 716 & & 726 & 710 \\
\hline 12 & 749 & 747 & & 768 & 750 \\
\hline 13 & 767 & 747 & & 771 & 760 \\
\hline 14 & 784 & 818 & & 786 & 780 \\
\hline 15 & & & & 921 & 890 \\
\hline 16 & & & & 964 & 940 \\
\hline 17 & & & & 984 & 980 \\
\hline 18 & & 1088 & & 1082 & 1060 \\
\hline 19 & 1103 & 1090 & 1106 & 1120 & 1110 \\
\hline 20 & 1198 & 1196 & 1193 & 1208 & 1190 \\
\hline 21 & 1237 & 1244 & 1227 & 1258 & 1220 \\
\hline 22 & 1340 & 1337 & 1335 & 1351 & 1320 \\
\hline 23 & 1423 & 1423 & 1408 & 1441 & 1420 \\
\hline 24 & 1475 & 1475 & 1472 & 1497 & 1480 \\
\hline 25 & 1540 & 1539 & 1521 & 1566 & 1550 \\
\hline 26 & 1602 & 1598 & 1588 & 1629 & 1590 \\
\hline 27 & 1659 & 1656 & 1647 & 1685 & 1660 \\
\hline 28 & 1730 & 1733 & 1736 & 1755 & 1750 \\
\hline 29 & & & 3037 & 3202 & 3140 \\
\hline 30 & & & 3092 & 3227 & 3120 \\
\hline 31 & 3457 & 3441 & 3460 & 3596 & 3510 \\
\hline 32 & 3474 & 3472 & 3477 & 3611 & 3510 \\
\hline 33 & 3575 & 3564 & 3610 & 3741 & 3580 \\
\hline MAE & & & $10(13)$ & $38(41)$ & $13(18)$ \\
\hline
\end{tabular}

MC SCIVR and DC SCIVR values are very similar and close to the experimental values as well. The MAE obtained by comparison with the experimental results is, respectively, 22 and 20 wavenumbers, which is pretty much the accuracy of other semiclassical simulations. Actually, also the MAE for the harmonic approximation at the same level of theory is quite similar. We think this good accuracy is accidental because higher level $a b$ initio harmonic estimates (see Table I of Ref. 30) provide frequencies which are systematically higher than B3LYP ones. Going back to the semiclassical results, we think that the DC SCIVR MAE is slightly smaller than the more accurate MC SCIVR one either because of a compensation of errors or because MC SCIVR has been pushed too close to the dimensional limit of the method. These results confirm that the main advantage of the DC SCIVR approach is given by its portability and applicability to higher-dimensional molecules by retaining a comparable accuracy to that one of the MC SCIVR.

Interestingly, MC SCIVR is able to recover some experimental aspects which are very hard to be reproduced, such as the exchange in energy levels between modes 7 and 8 . Namely, by employing normal mode or GVPT2 normal mode analysis (see, respectively, columns three and four in Table I) or VPT2 approaches (columns five and six of the same table), mode 7 results lower in energy than mode 8 , while according to the experimental assignment mode,

TABLE III. The same as Table II but for the hydroxycytosine isomer.

\begin{tabular}{|c|c|c|c|c|}
\hline Label & $\mathrm{Exp} / \mathrm{Ne}$ & Exp/Ar & Harmonic & DC SCIVR \\
\hline 8 & 553 & 557 & 560 & 540 \\
\hline 9 & 525 & 520 & 563 & 540 \\
\hline 10 & 600 & 601 & 602 & 570 \\
\hline 11 & 711 & 710 & 721 & 730 \\
\hline 12 & 784 & 751 & 790 & 790 \\
\hline 13 & 796 & 781 & 794 & 770 \\
\hline 14 & 809 & 807 & 818 & 830 \\
\hline 15 & 948 & 955 & 986 & 970 \\
\hline 16 & 948 & 955 & 993 & 970 \\
\hline 17 & 989 & 980 & 997 & 980 \\
\hline 18 & 1085 & 1083 & 1092 & 1090 \\
\hline 19 & 1113 & 1110 & 1120 & 1100 \\
\hline 20 & 1198 & 1196 & 1241 & 1220 \\
\hline 21 & 1258 & 1257 & 1298 & 1270 \\
\hline 22 & 1338 & 1333 & 1342 & 1330 \\
\hline 23 & 1382 & 1379 & 1399 & 1380 \\
\hline 24 & 1441 & 1439 & 1459 & 1440 \\
\hline 25 & 1495 & 1496 & 1517 & 1490 \\
\hline 26 & 1576 & 1575 & 1606 & 1570 \\
\hline 27 & 1592 & 1589 & 1630 & 1610 \\
\hline 28 & 1625 & 1622 & 1656 & 1640 \\
\hline 29 & & & 3165 & 3080 \\
\hline 30 & & & 3207 & 3090 \\
\hline 31 & 3461 & 3446 & 3596 & 3480 \\
\hline 32 & 3575 & 3564 & 3736 & 3600 \\
\hline 33 & 3618 & 3592 & 3770 & 3670 \\
\hline MAE & & & $41(36)$ & $16(18)$ \\
\hline
\end{tabular}




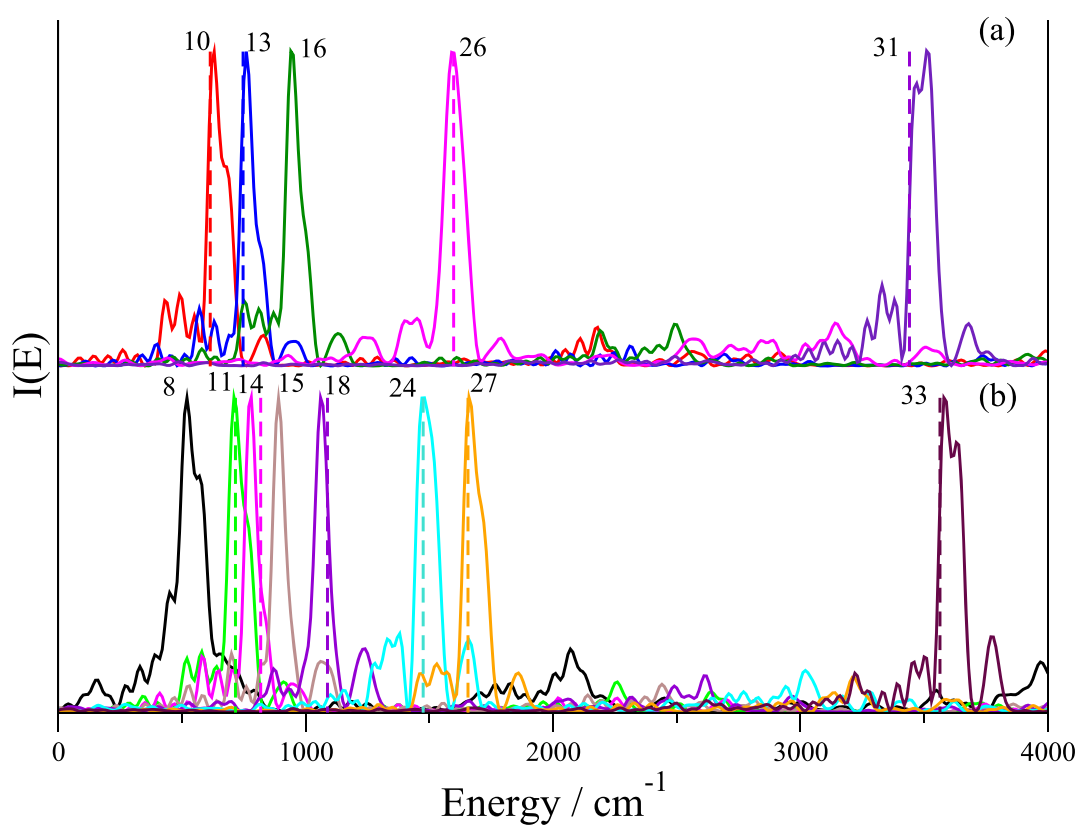

FIG. 3. Panel (a) and (b): The same as in Fig. 2 but for the 20-dimensional subspace of the oxocytosine isomer. Labels of the energy levels follow the nomenclature reported in Table II.

7 is slightly higher in energy than mode 8. MC SCIVR estimates agree with the experimental picture by locating mode 7 at $552 \mathrm{~cm}^{-1}$ and mode 8 at $544 \mathrm{~cm}^{-1}$. We believe this peak inversion can be reproduced only by including the relevant anharmonic effects experienced by semiclassical trajectories far from the equilibrium configuration.

Figure 2 reports the spectrum of the 17-dimensional uracil subspace. Each fundamental excitation was obtained by tailoring the reference state $|\chi\rangle$ of the semiclassical integrand according to Eq. (10). Vertical dashed lines in the figures are centered at the available experimental levels. The peaks are labeled according to the first column of Table I, and the values are reported in the same table.

In summary, our results for uracil molecule show that all relevant anharmonic effects can be reproduced and that the full dimensional MC SCIVR calculations lead to accurate results. DC SCIVR

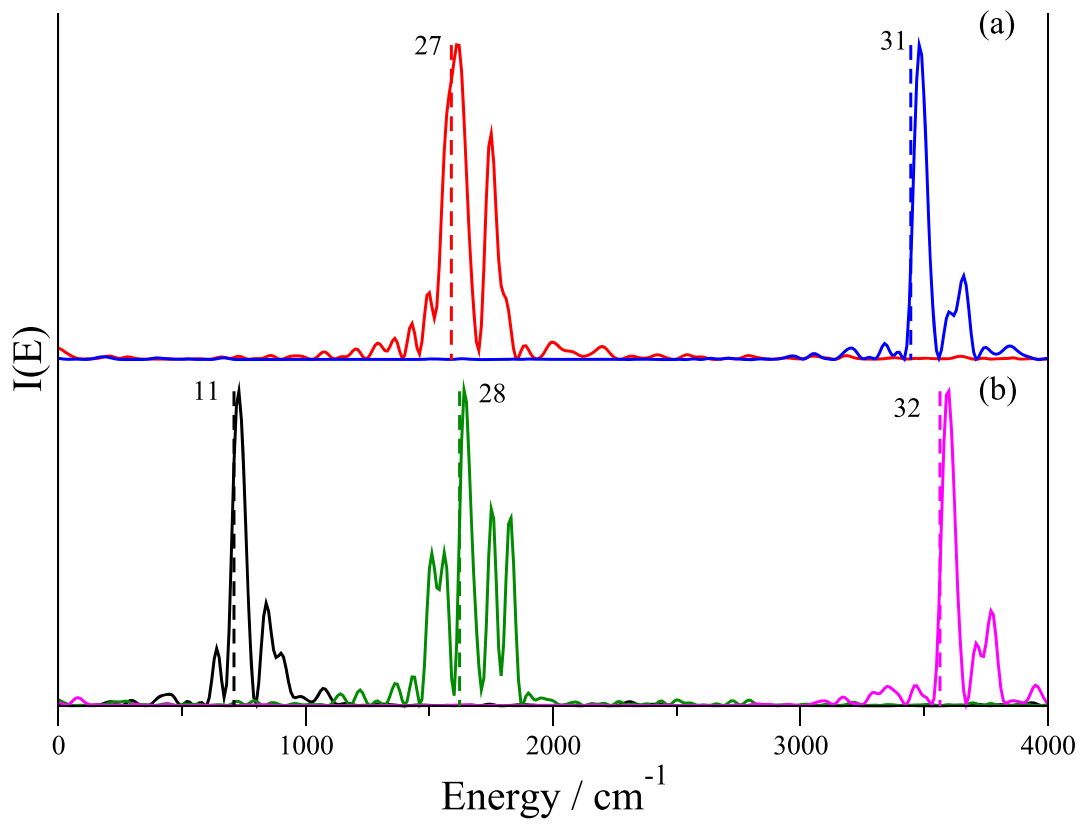

FIG. 4. Panel (a) and (b): The same as Fig. 3 but for the 11-dimensional hydroxycytosine isomer subspaces. Missing peaks in the figure are those with initial momenta set to 0 . Labels of the energy levels follow the nomenclature reported in Table III. 
results are comparable and enough accurate to pursue other nucleobase investigation, where full-dimensional MC SCIVR calculations cannot be longer afforded.

\section{B. Cytosine}

The next nucleobase we treat is cytosine, which is constituted by a pyrimidine ring. It differs from uracil by the presence of the amino group in place of an oxygen atom. This molecule is made by 13 atoms, resulting into 33 vibrational degrees of freedom. In addition to the increased dimension, the vibrational spectroscopic investigation gets complicated by the tautomerism between the oxocytosine and the hydroxycytosine. Both forms are spectroscopic relevant, given the very small minimum energy difference of few kilojoule/mole. ${ }^{23}$ This difference is $2.1 \mathrm{~kJ} / \mathrm{mol}$ at our level of DFT theory ${ }^{21}$ and in favor of the oxo form. Thus, we perform molecular dynamics simulations of both tautomers by running two classical trajectories, one starting from the oxocytosine isomer and the other from the hydroxycytosine one [respectively, panels (b) and (c) of Fig. 1]. We observe that there is no isomeric change along the entire simulation time of 25000 a.u. For this nucleobase, we observe the presence of a strong coupling between hindered rotations and other vibrational modes. For this reason, the initial kinetic energy of the first seven lowest frequency vibrational modes is set to zero since rotational contributions would jeopardize the numerical convergence of the spectra. This strategy is similar to what has been done in previous semiclassical calculations, ${ }^{70,71}$ and it does not represent a bias since the initial kinetic energy of the trajectory is reduced by only $5 \%$ below the harmonic $Z P E$ value, which is obviously in excess with respect to the actual ZPE. The columns of Tables II and III, labeled "Harmonic," report the harmonic frequencies of both isomers. We observe from the experimental values that most of the fundamentals of the two isomers are very similar in energy and this can be seen already at the harmonic level. A common strategy to discriminate between the two isomers is to look at the region around 3400-3700 wavenumbers. In that region are present for both isomers the symmetric and asymmetric $\mathrm{NH}_{2}$ stretches, around $3500-3600 \mathrm{~cm}^{-1}$. Additionally the oxo form shows a peak around $3450 \mathrm{~cm}^{-1}$ corresponding to the $\mathrm{NH}$ stretching, which is missing in hydroxycitosine spectrum that instead shows a signal above $3600 \mathrm{~cm}^{-1}$, due to the $\mathrm{OH}$ stretching. This trend is well reproduced by the DC SCIVR results that can effectively discriminate between the two tautomers. Tables II and III report the DC SCIVR computed energy levels and show the comparison with available experimental data and other theoretical results. The full dimensional vibrational space is divided into subspaces using a threshold value of $\varepsilon=7 \times 10^{-7}$ for Hessian elements. This choice generates for the oxocytosine one 20 dimensional subspace, leaving all the others monodimensional. In the case of hydroxycytosine, the threshold is fixed at $\varepsilon=2 \times 10^{-6}$ and the full-dimensional space is fragmented into one 11-dimensional, one 7-dimensional, and all remaining monodimensional. We observe that for both isomers, the agreement with the experiment is very strict, giving a MAE around $18 \mathrm{~cm}^{-1}$ for both systems, even if there are some frequencies which are occasionally quite off the mark. Harmonic estimates show a double MAE deviation.

Figure 3 shows DC SCIVR spectra for a 20-dimensional subspace of the oxocytosine isomer, while Fig. 4 reports the computed spectra for a 11-dimensional subspaces of the hydroxycytosine isomer. We observe that the number of peaks in Figs. 3 and 4 is less than the subspace-dimension since such subspace contains one low index mode (1-7). These figures show neat spectroscopic signals with several overtones reproduced, especially Fig. 4.

So far we have looked at the lowest dimensional couple of nucleobases made by a pyrimidine ring. Our results agree with

TABLE IV. Table for the vibrational frequencies of thymine. First column reports the label of the excitation. Columns two and three are for gas phase spectra of the isolated ${ }^{15}$ and Ar-tagged thymine. ${ }^{18,19}$ Column four reports the harmonic estimate at the level of B3LYPlaug-cc-pvdz, and the last column reports our computed DC SCIVR energy levels at the same level of theory. MAE is reported on the last row in comparison with both series of experiments. Values are reported in $\mathrm{cm}^{-1}$.

\begin{tabular}{|c|c|c|c|c|}
\hline Mode & Gas & Gas/Ar & Harmonic & DC SCIVR \\
\hline 1 & & & 130 & 110 \\
\hline 2 & & & 162 & 150 \\
\hline 3 & & & 168 & 150 \\
\hline 4 & & 280 & 281 & 290 \\
\hline 5 & & & 302 & 310 \\
\hline 6 & & 391 & 394 & 380 \\
\hline 7 & & 394 & 407 & 390 \\
\hline 8 & 462 & 455 & 461 & 460 \\
\hline 9 & & 540 & 546 & 530 \\
\hline 10 & 541 & 545 & 583 & 570 \\
\hline 11 & & & 603 & 600 \\
\hline 12 & 689 & 662 & 700 & 670 \\
\hline 13 & & 727 & 735 & 710 \\
\hline 14 & 755 & 754 & 757 & 750 \\
\hline 15 & 767 & 764 & 779 & 770 \\
\hline 16 & 804 & 800 & 802 & 780 \\
\hline 17 & 885 & 890 & 911 & 900 \\
\hline 18 & 963 & 959 & 962 & 950 \\
\hline 19 & & 1005 & 1018 & 1000 \\
\hline 20 & 1031 & 1046 & 1060 & 1040 \\
\hline 21 & 1078 & 1087 & 1153 & 1120 \\
\hline 22 & 1178 & 1184 & 1196 & 1170 \\
\hline 23 & & 1221 & 1232 & 1190 \\
\hline 24 & & 1357 & 1367 & 1380 \\
\hline 25 & & 1395 & 1397 & 1360 \\
\hline 26 & 1393 & 1389 & 1408 & 1390 \\
\hline 27 & 1409 & 1405 & 1419 & 1380 \\
\hline 28 & & 1437 & 1448 & 1430 \\
\hline 29 & & 1451 & 1473 & 1460 \\
\hline 30 & 1463 & 1472 & 1497 & 1460 \\
\hline 31 & 1668 & 1684 & 1700 & 1690 \\
\hline 32 & 1725 & 1712 & 1741 & 1750 \\
\hline 33 & 1772 & 1768 & 1784 & 1760 \\
\hline 34 & & 2855 & 3038 & 2955 \\
\hline 35 & 2941 & 2940 & 3097 & 2970 \\
\hline 36 & 2984 & 2971 & 3121 & 2990 \\
\hline 37 & 3076 & 2992 & 3202 & 3080 \\
\hline 38 & 3437 & 3434 & 3588 & 3470 \\
\hline 39 & 3484 & 3485 & 3632 & 3530 \\
\hline MAE & & & $48(38)$ & $17(21)$ \\
\hline
\end{tabular}




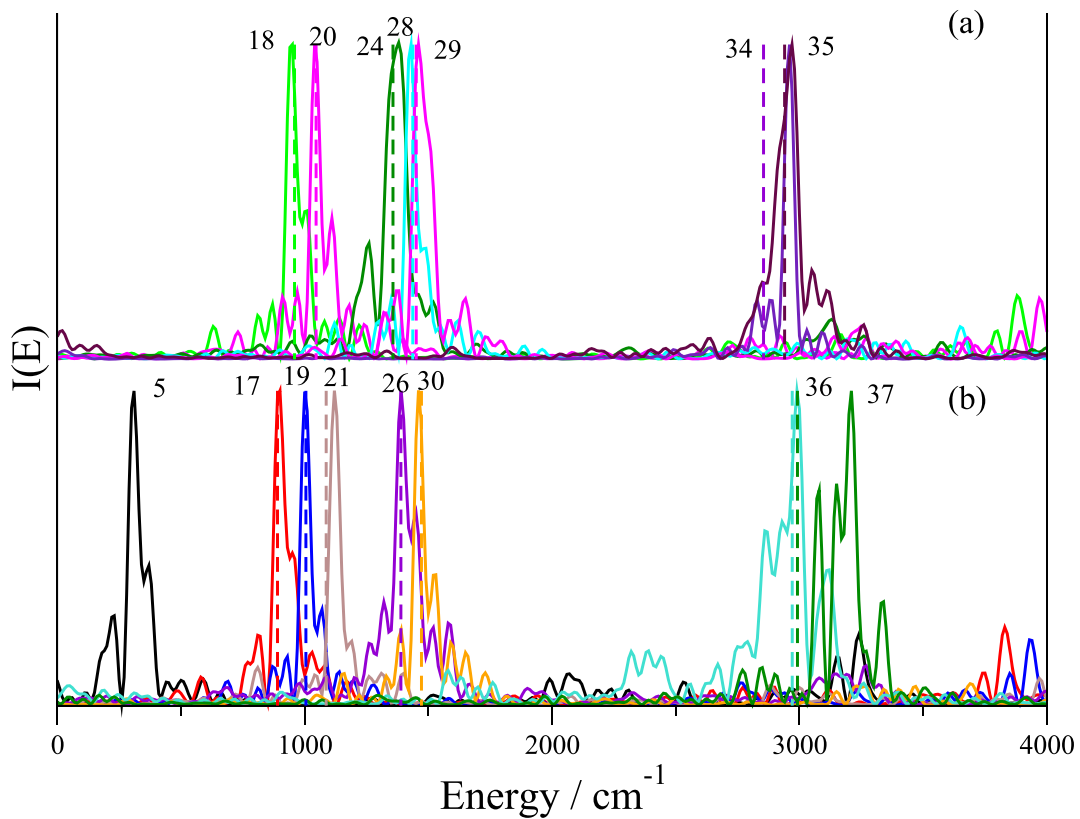

FIG. 5. Panel (a) and (b): The same as in Fig. 2 but for the 18-dimension subspace of thymine. Labels of the energy levels follow the nomenclature reported in Table IV. experiments with an average deviation of 15-20 wavenumbers, in line with previous semiclassical simulations, and are comparable with other theoretical methods. In Sec. III C, we look at thymine, the last pyrimidine-based nucleobase.

\section{Thymine}

Thymine is the highest dimension pyrimidine nucleobase. The structure is similar to uracil, where one hydrogen atom is substituted by a methyl group. Because of their resemblance, thymine and uracil show some similarities in biological processes and both link to adenine. The molecule is made of 15 atoms leading to 39 vibrational degrees of freedom. We calculate the vibrational spectra using classical trajectories starting from the global minimum structure, as we have done for uracil in Sec. III B, since the hydroxy tautomer minimum is about $44 \mathrm{~kJ} / \mathrm{mol}$ less stable, ${ }^{20}$ a value similar to the one between uracil tautomers $(45 \mathrm{~kJ} / \mathrm{mol}){ }^{21}$ This is expected given their structural similarity. The geometry of the molecule at

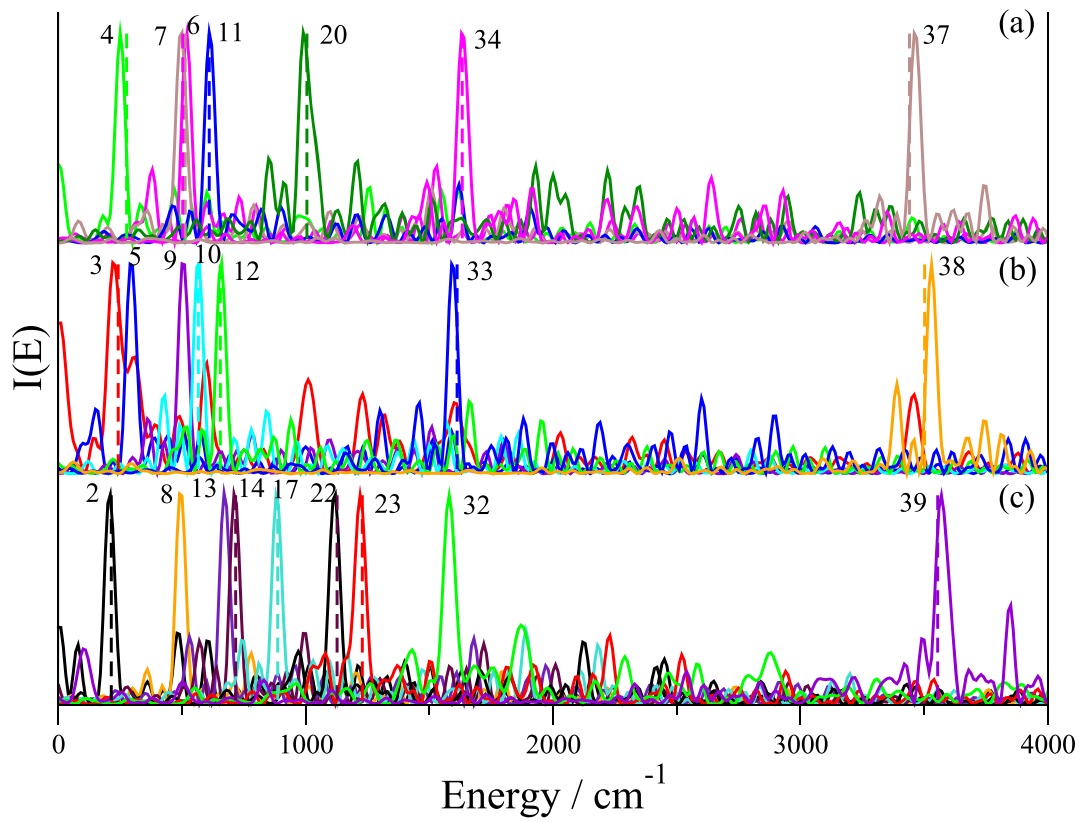

FIG. 6. Panel (a)-(c): The same as in Fig. 2 but for the 23-dimensional subspace of adenine. Frequency labels follow the nomenclature reported in Table IV. 
equilibrium configuration is reported in Panel (d) of Fig. 1. The Hessian partitioning method leads to a 18-dimensional subspace, a seven-dimensional one and all the others are mono-dimensional, when employing a threshold parameter $\varepsilon=8 \times 10^{-7}$. DC SCIVR frequencies are calculated for each subspace.

Table IV reports the vibrational frequencies at different levels of calculation and compares them with the experimental ones. In the table, DC SCIVR values are compared with the experimental energies of gas phase isolated and Ar-tagged thymine. We also report modes 1-3 even if no experimental data are available at the best of our knowledge. From the table, we observe that the MAE of DC SCIVR estimates is equal to 17 and 21 wavenumbers in comparison with gas phase and Ar-tagged levels, respectively, which is less than half the harmonic estimates. These values are in line once again with the typical semiclassical accuracy and are comparable with what was found from previous pyrimidine bases.

Figure 5 shows the computed DC SCIVR 18 dimension spectra, and one can note how the thymine fingerprint region around 1000 wavenumbers is very crowded. However, by using Eq. (10), we are able to selectively report each peak, as shown in Fig. 5. In this way, it is possible to resolve and attribute peaks which would be otherwise hardly distinguishable, since they are within few wavenumbers in energy, as in the case of modes 18-20 and $28-30$.

\section{Adenine}

Now, we move our attention to the remaining nucleobase, adenine. Adenine is a nucleobase made of a purine ring, composed of a pyrimidine condensed with an imidazole. The molecular structure at the equilibrium configuration is reported in panel (e) of Fig. 1, showing 15 atoms and resulting into 39 vibrational degrees of freedom, the same as thymine. In this study, we focus our attention only on the global minimum structure since previous works suggested that the other tautomers of the molecule are located around $30 \mathrm{~kJ} / \mathrm{mol}$ above the global minimum, an amount of energy difference that is close to that of thymine and uracil, rather than cytosine.

The 39 vibrational degree of freedom space is separated into one 23-dimensional, two bidimensional, and all other are monodimensional subspaces, by employing a threshold parameter for the Hessian method equal to $\varepsilon=9 \times 10^{-7}$. Figure 6 reports the spectrum of the highest dimensional subspace for this molecule.

Once again, our reference state choice according to Eq. (10) helps us to resolve different peaks which are very close in energy, like modes $37-39$ or 32-34. Figure 6 presents several overtone peaks, in comparison with previous ones. We believe this is due to the presence of the $\mathrm{NH}_{2}$ hindered rotation, which gets easily vibrational excited during the dynamics. Nevertheless, the full width at half maximum (FWHM), which denotes the peak definition, is quite small. For a more detailed comparison with experiments, Table $\mathrm{V}$ reports the computed energy levels and shows the comparison with available theoretical and experimental results, measured in the isolated gas phase and Ar matrix. The average deviation of the DC SCIVR results from both experiments is quite small, only 16 and 14 wavenumbers, almost three times more accurate than harmonic estimates. This accuracy is comparable with previous system investigations and with PT2 estimates. Overall, the DC SCIVR spectrum of adenine is further proof of the invariance of accuracy of our approach with the increase in nucleobase dimensionality. We believe that the investigation of this purine nucleobase strengthens previous considerations about pyrimidine-based nucleobases, in terms of spectroscopic quality and accuracy of the energy levels, when compared with the experiments.

TABLE V. Vibrational frequencies for adenine. First column reports the label of the excitation. Columns two and three are for gas phase spectra of isolated ${ }^{15}$ and Ar-tagged adenine. ${ }^{16}$ Column four reports the Perturbation Theory (PT2) energy levels. ${ }^{33}$ Column five report the harmonic estimate at the level of B3LYP/aug-cc-pvdz, and the last column reports our computed DC SCIVR energy levels at the same level of theory. MAE is reported on the last row, where the value in parentheses refers to the experiment in the Ar matrix. Values are reported in $\mathrm{cm}^{-1}$.

\begin{tabular}{|c|c|c|c|c|c|}
\hline Mode & Gas & Gas/Ar & PT2 & Harmonic & DC SCIVR \\
\hline 1 & 162 & & 139 & 168 & 160 \\
\hline 2 & & 214 & 181 & 210 & 210 \\
\hline 3 & 244 & 242 & 209 & 267 & 220 \\
\hline 4 & 270 & 276 & 276 & 279 & 250 \\
\hline 5 & & & 299 & 302 & 290 \\
\hline 6 & 506 & 503 & 491 & 512 & 520 \\
\hline 7 & 515 & 513 & 516 & 529 & 500 \\
\hline 8 & & & 518 & 531 & 490 \\
\hline 9 & & & 529 & 542 & 500 \\
\hline 10 & 563 & 566 & 570 & 583 & 570 \\
\hline 11 & 600 & 610 & 610 & 617 & 610 \\
\hline 12 & 650 & 655 & 655 & 668 & 660 \\
\hline 13 & & & 677 & 688 & 670 \\
\hline 14 & & 717 & 717 & 723 & 710 \\
\hline 15 & 801 & 802 & 811 & 815 & 800 \\
\hline 16 & 847 & 848 & 846 & 858 & 840 \\
\hline 17 & & 887 & 885 & 896 & 880 \\
\hline 18 & 926 & 927 & 931 & 942 & 930 \\
\hline 19 & 957 & 958 & 969 & 979 & 960 \\
\hline 20 & & 1005 & 1018 & 1015 & 990 \\
\hline 21 & 1065 & 1061 & 1054 & 1076 & 1050 \\
\hline 22 & 1126 & 1127 & 1125 & 1141 & 1120 \\
\hline 23 & 1234 & 1229 & 1230 & 1242 & 1220 \\
\hline 24 & & 1246 & 1243 & 1263 & 1250 \\
\hline 25 & 1280 & 1290 & 1291 & 1332 & 1300 \\
\hline 26 & 1326 & 1328 & 1325 & 1356 & 1330 \\
\hline 27 & 1346 & 1345 & 1338 & 1365 & 1330 \\
\hline 28 & & 1389 & 1376 & 1414 & 1390 \\
\hline 29 & 1415 & 1419 & 1406 & 1432 & 1400 \\
\hline 30 & 1468 & 1474 & 1466 & 1498 & 1485 \\
\hline 31 & & 1482 & 1481 & 1512 & 1500 \\
\hline 32 & & & 1577 & 1607 & 1580 \\
\hline 33 & & 1612 & 1591 & 1635 & 1590 \\
\hline 34 & 1625 & 1633 & 1616 & 1660 & 1630 \\
\hline 35 & & 3041 & 3049 & 3172 & 3070 \\
\hline 36 & 3061 & 3057 & 3102 & 3245 & 3160 \\
\hline 37 & 3434 & 3441 & 3432 & 3588 & 3460 \\
\hline 38 & 3501 & 3502 & 3497 & 3641 & 3530 \\
\hline 39 & 3552 & 3555 & 3539 & 3727 & 3570 \\
\hline MAE & & & $10(9)$ & $42(38)$ & $16(14)$ \\
\hline
\end{tabular}




\section{SUMMARY AND CONCLUSIONS}

In this paper, we have presented a semiclassical investigation of the vibrational features of uracil, cytosine, thymine, and adenine nucleobases. The investigation on uracil has shown that MC SCIVR energy levels are on average around 20 wavenumbers away from experimental levels, a typical value for semiclassical spectroscopic calculations. Then, the DC SCIVR method leads to values very close to the full-dimensional MC SCIVR ones, proving its reliability for the calculation of similar systems. Moving to higher dimensional nucleobases, DC SCIVR energy levels of cytosine retain their typical accuracy with respect to the experimental results. Despite the presence of more than one comparable minimum, the method still reproduces the spectra of different isomers retaining the standard accuracy of semiclassical simulations. Then, we focus on the last pyrimidine molecule, thymine, which is the highest dimensional nucleobase of this type. We have found that DC SCIVR retains its accuracy despite the increased dimensionality. Similar considerations hold for the adenine case, where the MAE is around 15 wavenumbers and comparable with PT2 estimates. Overall, we always find the accuracy of the DC SCIVR method to be comparable to other state of the art theoretical spectroscopy methods.

These outcomes are promising for a future exploitation of the method. Since the accuracy is seemingly insensitive to the increase in the molecule dimensionality, we will exploit DC SCIVR also to study more complex systems, like nucleotides and nucleobase pairs. This will possibly pave the way toward the assessment of important structural features of nucleoacids that lead to the formation of secondary and tertiary structures.

\section{ACKNOWLEDGMENTS}

The authors thank Dr. Riccardo Conte for useful discussions. The authors acknowledge support from the European Research Council (ERC) under the European Union's Horizon 2020 research and innovation programme (Grant Agreement No. 647107SEMICOMPLEX-ERC-2014-CoG). The authors acknowledge also CINECA (Italian Supercomputing Center) for providing high performance computational resources under the IscraB grant (QUASP). All authors thank Università degli Studi di Milano for further computational time at CINECA.

\section{REFERENCES}

${ }^{1}$ J. D. Watson and F. H. Crick, Nature 171, 964 (1953).

${ }^{2}$ V. Gabelica, Nucleic Acids in the Gas Phase (Springer, 2016).

${ }^{3}$ R. Rein, M. Shibata, R. Garduno-Juarez, and T. Kieber-Emmons, Structure and Dynamics: Nucleic Acids and Proteins (Adenine Press, New York, 1983), pp. 269-288.

${ }^{4}$ V. Danilov and G. Kventsel, Electronic Representations in the Point Mutation Theory (Naukova Dumka, Kiev, 1971).

${ }^{5}$ C. Colominas, F. J. Luque, and M. Orozco, J. Am. Chem. Soc. 118, 6811 (1996).

${ }^{6}$ R. D. Wells, Trends Biochem. Sci. 32, 271 (2007).

${ }^{7}$ M.-P. Gaigeot and M. Sprik, J. Phys. Chem. B 107, 10344 (2003).

${ }^{8}$ M.-P. Gaigeot and M. Sprik, J. Phys. Chem. B 108, 7458 (2004).

${ }^{9}$ P. López-Tarifa, M.-P. Gaigeot, R. Vuilleumier, I. Tavernelli, M. Alcamí, F. Martín, M.-A. Hervé du Penhoat, and M.-F. Politis, Angew. Chem. 125, 3242 (2013).

${ }^{10}$ T. Gustavsson, Á. Bányász, E. Lazzarotto, D. Markovitsi, G. Scalmani, M. J. Frisch, V. Barone, and R. Improta, J. Am. Chem. Soc. 128, 607 (2006).
${ }^{11}$ M. Baer, D. Marx, and G. Mathias, Angew. Chem., Int. Ed. 49, 7346 (2010).

${ }^{12}$ G. Mathias, S. D. Ivanov, A. Witt, M. D. Baer, and D. Marx, J. Chem. Theory Comput. 8, 224 (2011).

${ }^{13}$ O. Asvany, P. Kumar, B. Redlich, I. Hegemann, S. Schlemmer, and D. Marx, Science 309, 1219 (2005).

${ }^{14}$ A. M. Rijs and J. Oomens, Gas-Phase IR Spectroscopy and Structure of Biological Molecules (Springer, 2015), Vol. 364.

${ }^{15}$ P. Colarusso, K. Zhang, B. Guo, and P. F. Bernath, Chem. Phys. Lett. 269, 39 (1997).

${ }^{16}$ M. J. Nowak, L. Lapinski, J. S. Kwiatkowski, and J. Leszczyński, J. Phys. Chem. 100, 3527 (1996).

${ }^{17}$ D. Picconi, F. J. A. Ferrer, R. Improta, A. Lami, and F. Santoro, Faraday Discuss. 163, 223 (2013).

${ }^{18}$ M. Graindourze, J. Smets, T. Zeegers-Huyskens, and G. Maes, J. Mol. Struct. 222, 345 (1990).

${ }^{19}$ A. Leś, L. Adamowicz, M. J. Nowak, and L. Lapinski, Spectrochim. Acta, Part A 48, 1385 (1992).

${ }^{20}$ J. Rejnek, M. Hanus, M. Kabeláč, F. Ryjáček, and P. Hobza, Phys. Chem. Chem. Phys. 7, 2006 (2005).

${ }^{21}$ S. Tian, C. Zhang, Z. Zhang, X. Chen, and K. Xu, Chem. Phys. 242, 217 (1999).

${ }^{22}$ M. Y. Choi and R. E. Miller, J. Phys. Chem. A 111, 2475 (2007).

${ }^{23}$ J. S. Kwiatkowski and J. Leszczyński, J. Phys. Chem. 100, 941 (1996).

${ }^{24}$ E. Nir, C. Janzen, P. Imhof, K. Kleinermanns, and M. De Vries, J. Chem. Phys. 115, 4604 (2001).

${ }^{25}$ M. Y. Choi and R. E. Miller, J. Am. Chem. Soc. 128, 7320 (2006).

${ }^{26}$ M. Nowak, L. Lapinski, and J. Fulara, Spectrochim. Acta, Part A 45, 229 (1989).

${ }^{27}$ M. Mons, I. Dimicoli, F. Piuzzi, B. Tardivel, and M. Elhanine, J. Phys. Chem. A 106, 5088 (2002).

${ }^{28}$ M. Mons, F. Piuzzi, I. Dimicoli, L. Gorb, and J. Leszczynski, J. Phys. Chem. A 110, 10921 (2006).

${ }^{29}$ C. M. Marian, J. Phys. Chem. A 111, 1545 (2007).

${ }^{30}$ C. Puzzarini, M. Biczysko, and V. Barone, J. Chem. Theory Comput. 7, 3702 (2011).

${ }^{31}$ S. V. Krasnoshchekov, N. Vogt, and N. F. Stepanov, J. Phys. Chem. A 119, 6723 (2015).

${ }^{32}$ P. S. Thomas, T. Carrington, Jr., J. Agarwal, and H. F. Schaefer III, J. Chem. Phys. 149, 064108 (2018).

${ }^{33}$ M. Biczysko, P. Panek, and V. Barone, Chem. Phys. Lett. 475, 105 (2009).

${ }^{34}$ R. Wałęsa, T. Kupka, and M. A. Broda, Struct. Chem. 26, 1083 (2015).

${ }^{35}$ W. H. Miller and T. F. George, J. Chem. Phys. 56, 5637 (1972).

${ }^{36}$ W. H. Miller, Proc. Natl. Acad. Sci. U. S. A. 102, 6660 (2005).

${ }^{37}$ W. H. Miller, J. Chem. Phys. 53, 1949 (1970).

${ }^{38}$ E. Heller, J. Chem. Phys. 95, 9431 (1991).

${ }^{39}$ K. G. Kay, Annu. Rev. Phys. Chem. 56, 255 (2005).

${ }^{40}$ D. J. Tannor, Introduction to Quantum Mechanics (University Science Books, 2007).

${ }^{41}$ J. Shao and N. Makri, J. Phys. Chem. A 103, 7753 (1999).

${ }^{42}$ E. Pollak, "The semiclassical initial value series representation of the quantum propagator," in Quantum Dynamics of Complex Molecular Systems, edited by D. A. Micha and I. Burghardt (Springer Berlin Heidelberg, Berlin, Heidelberg, 2007), pp. 259-271.

${ }^{43}$ S. Zhang and E. Pollak, J. Chem. Theory Comput. 1, 345 (2005).

${ }^{44}$ D. V. Shalashilin and M. S. Child, J. Chem. Phys. 115, 5367 (2001).

${ }^{45}$ D. V. Shalashilin and M. S. Child, Chem. Phys. 304, 103 (2004).

${ }^{46}$ G. Tao, Theor. Chem. Acc. 133, 1448 (2014).

${ }^{47}$ J. Liu, Int. J. Quantum Chem. 115, 657 (2015).

${ }^{48}$ M. Church, S. V. Antipov, and N. Ananth, J. Chem. Phys. 146, 234104 (2017).

${ }^{49}$ Z. Ma and D. Coker, J. Chem. Phys. 128, 244108 (2008).

${ }^{50}$ J. Tatchen and E. Pollak, J. Chem. Phys. 130, 041103 (2009).

${ }^{51}$ M. Wehrle, M. Šulc, and J. Vaníček, J. Chem. Phys. 140, 244114 (2014).

${ }^{52} \mathrm{M}$. Wehrle, S. Oberli, and J. Vaníček, J. Phys. Chem. A 119, 5685 (2015).

${ }^{53}$ R. Conte, A. Aspuru-Guzik, and M. Ceotto, J. Phys. Chem. Lett. 4, 3407 (2013). 
${ }^{54}$ M. Ceotto, S. Atahan, G. F. Tantardini, and A. Aspuru-Guzik, J. Chem. Phys. 130, 234113 (2009).

${ }^{55}$ M. Ceotto, S. Atahan, S. Shim, G. F. Tantardini, and A. Aspuru-Guzik, Phys. Chem. Chem. Phys. 11, 3861 (2009).

${ }^{56}$ M. Buchholz, F. Grossmann, and M. Ceotto, J. Chem. Phys. 147, 164110 (2017).

${ }^{57}$ M. Buchholz, F. Grossmann, and M. Ceotto, J. Chem. Phys. 148, 114107 (2018).

${ }^{58}$ M. Buchholz, F. Grossmann, and M. Ceotto, J. Chem. Phys. 144, 094102 (2016).

${ }^{59}$ T. Begušić, A. Patoz, M. Šulc, and J. Vaníček, Chem. Phys. 515, 152 (2018).

${ }^{60}$ T. Begušić, J. Roulet, and J. Vaníček, J. Chem. Phys. 149, 244115 (2018).

${ }^{61}$ J. Vaníček, CHIMIA Int. J. Chem. 71, 283 (2017).

${ }^{62}$ M. Wehrle, M. Šulc, and J. Vaníček, CHIMIA Int. J. Chem. 65, 334 (2011).

${ }^{63}$ E. Zambrano, M. Šulc, and J. Vaníček, J. Chem. Phys. 139, 054109 (2013).

${ }^{64} \mathrm{M}$. Šulc and J. Vaníček, Mol. Phys. 110, 945 (2012).

${ }^{65}$ M. Buchholz, E. Fallacara, F. Gottwald, M. Ceotto, F. Grossmann, and S. D. Ivanov, Chem. Phys. 515, 231 (2018).

${ }^{66}$ M. Micciarelli, R. Conte, J. Suarez, and M. Ceotto, J. Chem. Phys. 149, 064115 (2018).

${ }^{67}$ M. Micciarelli, G. Fabio, R. Conte, and M. Ceotto, J. Chem. Phys. 150, 184113 (2019).

${ }^{68}$ M. Ceotto, S. Valleau, G. F. Tantardini, and A. Aspuru-Guzik, J. Chem. Phys. 134, 234103 (2011).

${ }^{69}$ M. Ceotto, G. Di Liberto, and R. Conte, Phys. Rev. Lett. 119, 010401 (2017).

${ }^{70}$ G. Di Liberto, R. Conte, and M. Ceotto, J. Chem. Phys. 148, 014307 (2018).

${ }^{71}$ G. Di Liberto, R. Conte, and M. Ceotto, J. Chem. Phys. 148, 104302 (2018).

${ }^{72}$ F. Gabas, G. Di Liberto, R. Conte, and M. Ceotto, Chem. Sci. 9, 7894 (2018).

${ }^{73}$ F. Gabas, R. Conte, and M. Ceotto, J. Chem. Theory Comput. 13, 2378 (2017).

${ }^{74}$ C. Plützer, I. Hünig, K. Kleinermanns, E. Nir, and M. S. de Vries, Chem. Phys. Chem. 4, 838 (2003).

${ }^{75}$ A. Abo-Riziq, L. Grace, E. Nir, M. Kabelac, P. Hobza, and M. S. de Vries, Proc. Natl. Acad. Sci. U. S. A. 102, 20 (2005).

${ }^{76}$ E. Nir, C. Janzen, P. Imhof, K. Kleinermanns, and M. De Vries, Phys. Chem. Chem. Phys. 4, 732 (2002).

${ }^{77}$ T. Fornaro, D. Burini, M. Biczysko, and V. Barone, J. Phys. Chem. A 119, 4224 (2015).

${ }^{78}$ E. Nir, C. Plützer, K. Kleinermanns, and M. De Vries, Eur. Phys. J. D 20, 317 (2002).
${ }^{79}$ R. P. Feynman and A. R. Hibbs, Quantum Mechanics and Path Integrals (McGraw-Hill, 1965).

${ }^{80}$ J. H. Van Vleck, Proc. Natl. Acad. Sci. U. S. A. 14, 178 (1928).

${ }^{81}$ E. J. Heller, J. Chem. Phys. 75, 2923 (1981).

${ }^{82}$ E. J. Heller, Acc. Chem. Res. 14, 368 (1981).

${ }^{83}$ M. F. Herman and E. Kluk, Chem. Phys. 91, 27 (1984).

${ }^{84}$ K. G. Kay, Chem. Phys. 322, 3 (2006).

${ }^{85}$ F. Grossmann and A. L. Xavier, Phys. Lett. A 243, 243 (1998).

${ }^{86}$ Y. Zhuang, M. R. Siebert, W. L. Hase, K. G. Kay, and M. Ceotto, J. Chem. Theory Comput. 9, 54 (2012).

${ }^{87}$ M. Ceotto, Y. Zhuang, and W. L. Hase, J. Chem. Phys. 138, 054116 (2013).

${ }^{88}$ K. G. Kay, J. Chem. Phys. 100, 4432 (1994).

${ }^{89}$ K. G. Kay, J. Chem. Phys. 100, 4377 (1994).

${ }^{90}$ K. G. Kay, J. Chem. Phys. 101, 2250 (1994).

${ }^{91}$ H. Wang, D. E. Manolopoulos, and W. H. Miller, J. Chem. Phys. 115, 6317 (2001).

${ }^{92}$ A. L. Kaledin and W. H. Miller, J. Chem. Phys. 118, 7174 (2003).

${ }^{93}$ A. L. Kaledin and W. H. Miller, J. Chem. Phys. 119, 3078 (2003).

${ }^{94}$ D. Tamascelli, F. S. Dambrosio, R. Conte, and M. Ceotto, J. Chem. Phys. 140, 174109 (2014).

${ }^{95}$ M. Ceotto, D. Dell'Angelo, and G. F. Tantardini, J. Chem. Phys. 133, 054701 (2010).

${ }^{96}$ G. Di Liberto and M. Ceotto, J. Chem. Phys. 145, 144107 (2016).

${ }^{97}$ M. Ceotto, G. F. Tantardini, and A. Aspuru-Guzik, J. Chem. Phys. 135, 214108 (2011).

${ }^{98}$ K. Hinsen and G. R. Kneller, Mol. Simul. 23, 275 (2000).

${ }^{99}$ B. B. Harland and P.-N. Roy, J. Chem. Phys. 118, 4791 (2003).

${ }^{100}$ M. Valiev, E. Bylaska, N. Govind, K. Kowalski, T. Straatsma, H. V. Dam, D. Wang, J. Nieplocha, E. Apra, T. Windus, and W. de Jong, Comput. Phys. Commun. 181, 1477 (2010).

${ }^{101}$ A. D. Becke, J. Chem. Phys. 98, 5648 (1993).

${ }^{102}$ X. Ma, G. Di Liberto, R. Conte, W. L. Hase, and M. Ceotto, J. Chem. Phys. 149, 164113 (2018).

${ }^{103}$ M. Szczesniak, M. Nowak, H. Rostkowska, K. Szczepaniak, W. B. Person, and D. Shugar, J. Am. Chem. Soc. 105, 5969 (1983).

${ }^{104}$ S. Chin, I. Scott, K. Szczepani, and W. B. Person, J. Am. Chem. Soc. 106, 3415 (1984). 